\title{
A New Approach of Functional Dependency in a Neutrosophic Relational Database Model
}

\author{
Soumitra De ${ }^{1^{*}}$ and Jaydev Mishra ${ }^{2}$ \\ ${ }^{1 \& 2}$ Assistant Professor, Department of Computer Science and Engineering, \\ Maulana Abul Kalam Azad University of Technology, West Bengal, India \\ *Corresponding Author \\ E-Mail: soumitra@cemk.ac.in, jsm@cemk.ac.in
}

\begin{abstract}
In order to model the imprecise and uncertain information, different classical relational data model have been studied in literature using vague set theory. However, neutrosophic set, as a generalized vague set, has more powerful ability to process fuzzy information than vague set. In this paper, we have proposed a neutrosophic relational database model and have defined a new kind of neutrosophic functional dependency (called $\alpha$-nfd) based on the $\alpha$-equality of tuples and the similarity measure of neutrosophic sets. Next, we present a set of sound neutrosophic inference rules which are similar to Armstrong's axioms for the classical case. Finally, partial $\alpha$-nfdand neutrosophic key have been studied with the new notion of $\alpha$-nfdand also tested.

Keywords:Neutro-sophicSet, Similarity Measure of Neutrosophic Data, Neutro-sophic Functional Dependency( $\alpha$-Nfd), Partial $\alpha$-Nfd, Neutro-sophic Key
\end{abstract}

\section{INTRODUCTION}

Real world information is very often imprecise in nature. Neutrosophic set theory, introduced by Smarandache in $2001[\mathrm{I}]$ has been widely used in literature [VIII, IX, X, IV, $\mathrm{XI}]$ to incorporate such imprecise data into classical relational databases.However, vague set theory was put forward by Gau and Buehrer [XVI] in 1993 as a more efficient tool to deal with uncertain data. A vague set, conceived as a generalization of the concept of fuzzy set, is a set of objects each of which has a grade of membership whose value is a continuous sub-interval of $[0,1]$.

A vague set $V$ which is characterized by a truth membership function $t_{V}$ and a false membership function $f_{V}$ where $t_{V}+f_{V} \leq$ 1 . In vague set certain portion i.e., $\left(1-t_{V}+f_{V}\right)$ is still undeterministic and also affect on taking decision. Now neutrosophic set has been introduced to deal with imprecise information in a more efficient manner than vague set theory using truth membership function $t_{v}$, indeterminacy function $i_{v}$ and a false membership function $f_{v}$. And the extended database model is then called a neutrosophic database model. However, compared to fuzzy and vague databases, much less research has been reported so far in the literature of neutrosophic database. Since data dependencies play an important role in any database design so, objective of this paper is to design the concepts of functional dependencies on neutrosophic database called neutrosophic functional dependency ( $\alpha$-nfd). The concept of $\alpha$-nfd is defined based on similarity measure of neutrosophic data which is again a new concept defined by the authors in this paper.This paper is organized as follows. Section 2 presents some basic knowledge about the neutrosophic set theory. Basic neutrosophic database concept is reported in section 3. In this section we also introduce new concepts of similarity measure of neutrosophic data, $\alpha$-equality of neutrosophic data, neutrosophic functional dependencies ( $\alpha$-nfd) between two sets of attributes. The said concepts are then used to define Armstrong's axioms and other inference rules,neutrosophic partial functional dependency and neutrosophic key. Finally in Section 4 we draw an overall conclusion of the work.

\section{BASICS OF NEUTROSOPHIC SET}

In this section, we introduce the new concept of neutrosophic set. Let $\mathrm{U}$ be the universe of discourse where an element of $\mathrm{U}$ is denoted by $\mathrm{u}$.

Definition 1: A neutrosophic set $\mathrm{X}$ on the universe of discourse $\mathrm{U}$ is characterized by three membership functions given by:

1. a truth membership function $t_{x}: U \rightarrow[0,1]$,

2. a false membership function $f_{x}: U \rightarrow[0,1]$ and

3. a indeterminacy membership function $i_{x}: U \rightarrow[0,1]$ such that $t_{x}(a)+f_{x}(a) \leq 1 \quad$ and $\quad t_{x}(a)+f_{x}(a)+i_{x}(a) \leq 2$ and iswritten as $X=\left\{\left\langle x,\left[t_{x}(a), i_{x}(a), f_{x}(a)\right]\right\rangle, a \in U\right\}$.

Definition 2:A Neutrosophic set ' $\mathrm{N}$ 'is an emptyneutrosophic set, denoted by $\phi$, if and only if its truth-membership function $t_{n}(u)=0$ indeterminacymembership function $\mathrm{i}_{\mathrm{n}}(u)=0$ and false-membership function $f_{n}(u)=1$ for all $u$ on $U$.

Definition 3: A Neutrosophic set ' $A$ ' is containedin another neutrosophic set ' $B$ ', written as $A \subseteq B$, if and only if, $t_{A} \leq t_{B}, i_{A} \leq \mathrm{l}_{\mathrm{B}}$ and $f_{A} \geq f_{B}$.

Definition 4:Two Neutrosophic sets ' $A$ ' and ' $B$ ' are equal, written as $A=B$, iff, $A \subseteq B$ and $B \subseteq A$, that is, $t_{A}=t_{B}, i_{A}=$ $\mathrm{i}_{\mathrm{B}}$ and $f_{A}=f_{B}$. 
Definition 5:The union of two neutrosophic sets $A$ and $B$ is a neutrosophic set $C$, denoted as $C=A \cup B$, whose truthmembership, indeterminacy-membership and falsemembership functions are related to those of $A$ and $B$ by $\mathrm{t}_{\mathrm{C}}$ $=\max \left(\mathrm{t}_{\mathrm{A}}, t_{B}\right), i_{C}=\min \left(\mathrm{i}_{\mathrm{A}}, \mathrm{i}_{\mathrm{B}}\right)$ and $f_{C}=\min \left(f_{A}, f_{B}\right)$.

Definition 6:The intersection of two Neutrosophic sets $A$ and $B$ is a neutrosophic set $C$, written as $C=A \cap B$, such that $\mathrm{t}_{\mathrm{C}}=\min \left(\mathrm{t}_{\mathrm{A}}, t_{B}\right), i_{C}=\max \left(i_{\mathrm{A}}, i_{\mathrm{B}}\right)$ and $f_{C}=\max \left(f_{A}, f_{B}\right)$.

\section{NEUTROSOPHIC RELATIONAL DATABASE MODEL}

Here, we make an attempt to extend the classical relational database model to incorporate neutrosophic data by means of neutrosophic set theory, which results in the Neutrosophic Relational Database model (NRDB).

TABLE I NEUTROSOPHIC RELATIONAL INSTANCE OF DE-QUERVAIN'S DISEASE RELATION

\begin{tabular}{|l|c|c|c|}
\hline \multicolumn{1}{|c|}{ Name } & Age & UricAcid(UA) & LiquidChromatography(LC) \\
\hline Asmith & 30 & $\{6.5<.92, .01, .03>\}$ & $\{6.9<.94, .01, .02>\}$ \\
\hline Bell & 22 & $\{7<.99, .0015, .01>\}$ & $\{6.5<.99, .012, .01>\}$ \\
\hline Jackson & 45 & $\{7.35<.9, .02, .04>\}$ & $\{6.8<.95, .01, .01>\}$ \\
\hline Bisop & 33 & $\{3.23<.71, .026, .12>\}$ & $\{7.5<.80, .015, .14>\}$ \\
\hline Ritesh & 40 & $\{7.1<.98, .01, .01>\}$ & $\{8.1<.74, .125, .22>\}$ \\
\hline Pradip & 58 & $\{6.85<.913, .012, .03>\}$ & $\{6.3<.96, .02, .01>\}$ \\
\hline Amlesh & 60 & $\{7.25<.97, .01, .02>\}$ & $\{7.4<.85, .015, .04>\}$ \\
\hline Nisitha & 52 & $\{6.4<.90, .02, .03>\}$ & $\{5.9<.91, .01, .06>\}$ \\
\hline Madhumita & 35 & $\{5.7<.76, .01, .22>\}$ & $\{6.4<.98, .001, .02>\}$ \\
\hline Neha & 43 & $\{7.15<.98, .001, .01>\}$ & $\{6.65<.97, .02, .03>\}$ \\
\hline
\end{tabular}

Example 1: Consider the neutrosophic relational instance $r$ over De-Quervain's Disease relation (NAME, UricAcid, LiquidChromatography) given above in Table 1. In $r$, UricAcid and LiquidChromatography are neutrosophic attributes. The first tuple in $r$ means the employee with $\mathrm{NAME}=$ "Asmith" has the uric acid of $\{6.5,<.92, .01, .03>\}$ and the LiquidChromatography of $\{6.9,<.94, .01, .02>\}$, which are neutrosophic sets. Here the neutrosophic data $\{6.5,<.92, .01, .03>\}$ means the evidence in favour of "The uric acid is 6.5 " is .92 , the indeterminacy part .01 and the evidence against it is .03 and so on.

\section{A. Similarity Measure of Neutrosophic Data}

There have been some studies in literature which discuss the topic concerning how to measure the degree of similarity between vague sets [II, III, V, VI, VII]. This similarity measure did not fit well in some cases. We have introduced a new similarity measure between neutrosophic sets [XII, $\mathrm{XIII}, \mathrm{XIV}, \mathrm{XV}]$ which turned out to be more reasonable in more general cases which have been used in the present work which is defined as follows:

Definition 1:Let $x$ and $y$ be any two neutrosophic values such that $x=\left[t_{x}, i_{x}, f_{x}\right]$ and $y=\left[t_{y}, i_{y}, f_{y}\right]$ where

$0 \leq t_{x} \leq 1,0 \leq i_{x} \leq 1,0 \leq f_{x} \leq 1$ and $0 \leq t_{y} \leq 1,0 \leq i_{y} \leq 1,0 \leq f_{y} \leq 1$

with $0 \leq t_{x}+f_{x} \leq 1,0 \leq t_{y}+f_{y} \leq 1,0 \leq t_{x}+i_{x}+f_{x} \leq 2$,

$0 \leq t_{y}+i_{y}+f_{y} \leq 2$
Now the similarity measure between two neutrosophic data denoted by $S E(x, y)$ is defined as follows

$$
S E(x, y)=\sqrt{1-\frac{\left|\left(t_{x}-t_{y}\right)-\left(i_{x}-i_{y}\right)-\left(f_{x}-f_{y}\right)\right|}{3}\left(1-\left|\left(t_{x}-t_{y}\right)+\left(i_{x}-i_{y}\right)+\left(f_{x}-f_{y}\right)\right|\right)}
$$

\section{B. Neutrosophic Functional Dependency}

In this paper, similar to classical functional dependency, we define a new notion of neutrosophic functional dependency (called $\alpha$-nfd) based on the concept of $\alpha$-equality of neurosophic tuples which plays important role in designing neutrosophic database.Next, we present a set of neutrosophic inference rules which are similar to Armstrong's axioms for the classical case.

Definition 2:Let $r(R)$ be a neutrosophic relation on the relational schema $R\left(A_{1}, A_{2}, \ldots \ldots, A_{n}\right)$. Let $t_{1}$ and $t_{2}$ be any two neutrosophic tuples in $r$. Let $\alpha \in[0,1]$ be a threshold or choice parameter, predefined by the database designer, and $X=\left\{A_{1}, A_{2}, \ldots \ldots, A_{k}\right\} \subseteq R$. Then the neutrosophic tuples $t_{1}$ and $t_{2}$ are said to be $\alpha$-equal on $X$ if $\operatorname{SE}\left(t_{1}\left[A_{i}\right], t_{2}\left[A_{i}\right]\right) \geq$ $\alpha \forall i=1,2,3 \ldots . . k$. We denote this equality by the notation $t_{1}[X](N E)_{\alpha} t_{2}[X]$. The following proposition is straightforward from the above definition.

Proposition 1:If $0 \leq \alpha_{2} \leq \alpha_{1} \leq 1$, then

$t_{1}[X](N E)_{\alpha_{1}} t_{2}[X] \Rightarrow t_{1}[X](N E)_{\alpha_{2}} t_{2}[X]$

Definition 3:Let $X, Y \subset R=\left\{A_{l}, A_{2} \ldots A_{n}\right\}$. Choose a threshold value $\alpha \in[0,1]$. Then a neutrosophic functional dependency ( $\alpha$-nfd), denoted by $X \stackrel{n f d}{\longrightarrow} Y$ is said to exist 
if, whenever $t_{1}[X](N E)_{\alpha} t_{2}[X]$, it is also the case that $t_{1}[Y](N E)_{\alpha} t_{2}[Y]$.It may be read as " $X$ neutrosophic functionally determines $Y$ at $\alpha$-level". In another way, " $Y$ is neutrosophic functionally determined by $X$ at $\alpha$-level". The following proposition for $\alpha$-nfd is straightforward.

Proposition 2:If $0 \leq \alpha_{2} \leq \alpha_{1} \leq 1$, then

$$
X \underset{\alpha_{1}}{\stackrel{n f f}{\longrightarrow}} Y \Rightarrow X \underset{\alpha_{2}}{\stackrel{n f d}{\longrightarrow}} Y
$$

Example 2:Consider the neutrosophic relational instance $r$

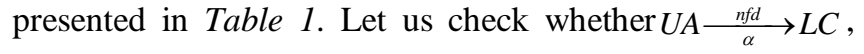
holds to a certain $\alpha$ - level of choice or not. Using the Definition 3.1 we have calculated $S E\left(t_{p}[U A], t_{q}[U A]\right)$ and $S E\left(t_{p}[L C], t_{q}[L C]\right)$ for every pair of tuples $t_{p}$ and $t_{q}$ and the results are shown below in Table 2 and Table 3 respectively.

TABLE II SIMILARITY MEASURE FOR UA

\begin{tabular}{|c|c|c|c|c|c|c|c|c|c|c|}
\hline & $\mathbf{t}_{\mathbf{1}}$ & $\mathbf{t}_{\mathbf{2}}$ & $\mathbf{t}_{\mathbf{3}}$ & $\mathbf{t}_{\mathbf{4}}$ & $\mathbf{t}_{\mathbf{5}}$ & $\mathbf{t}_{\mathbf{6}}$ & $\mathbf{t}_{\mathbf{7}}$ & $\mathbf{t}_{\mathbf{8}}$ & $\mathbf{t}_{\mathbf{9}}$ & $\mathbf{t}_{\mathbf{1 0}}$ \\
\hline $\mathrm{t}_{1}$ & 1 & .93 & .99 & .89 & .95 & .99 & .97 & .99 & .93 & .97 \\
\hline $\mathrm{t}_{2}$ & .93 & 1 & .97 & .91 & .99 & .98 & .99 & .98 & .93 & .99 \\
\hline $\mathrm{t}_{3}$ & .99 & .97 & 1 & .9 & .96 & .99 & .96 & .99 & .93 & .88 \\
\hline $\mathrm{t}_{4}$ & .89 & .91 & .9 & 1 & .86 & .92 & .87 & .81 & .93 & .86 \\
\hline $\mathrm{t}_{5}$ & .95 & .99 & .96 & .86 & 1 & .96 & .99 & .96 & .92 & .99 \\
\hline $\mathrm{t}_{6}$ & .99 & .98 & .99 & .92 & .96 & 1 & .97 & .99 & .94 & .97 \\
\hline $\mathrm{t}_{7}$ & .97 & .99 & .96 & .87 & .99 & .97 & 1 & .96 & .77 & .99 \\
\hline $\mathrm{t}_{8}$ & .99 & .98 & 1 & .81 & .96 & .99 & .96 & 1 & .93 & .96 \\
\hline $\mathrm{t}_{9}$ & .93 & .93 & .93 & .93 & .92 & .94 & .77 & .93 & 1 & .92 \\
\hline $\mathrm{t}_{10}$ & .97 & .99 & .88 & .86 & .99 & .97 & .99 & .96 & .92 & 1 \\
\hline
\end{tabular}

TABLE III SIMILARITY MEASURE FOR LC

\begin{tabular}{|c|c|c|c|c|c|c|c|c|c|c|}
\hline & $\mathbf{t}_{\mathbf{1}}$ & $\mathbf{t}_{\mathbf{2}}$ & $\mathbf{t}_{\mathbf{3}}$ & $\mathbf{t}_{\mathbf{4}}$ & $\mathbf{t}_{\mathbf{5}}$ & $\mathbf{t}_{\mathbf{6}}$ & $\mathbf{t}_{\mathbf{7}}$ & $\mathbf{t}_{\mathbf{8}}$ & $\mathbf{t}_{\mathbf{9}}$ & $\mathbf{t}_{\mathbf{1 0}}$ \\
\hline $\mathrm{t}_{1}$ & 1 & .96 & .99 & .95 & .85 & .98 & .94 & .98 & .97 & .96 \\
\hline $\mathrm{t}_{2}$ & .96 & 1 & .97 & .87 & .86 & .98 & .91 & .96 & .99 & .98 \\
\hline $\mathrm{t}_{3}$ & .99 & .97 & 1 & .94 & .85 & .99 & .94 & .97 & .98 & .97 \\
\hline $\mathrm{t}_{4}$ & .95 & .87 & .94 & 1 & .89 & .97 & .96 & .95 & .92 & .93 \\
\hline $\mathrm{t}_{5}$ & .85 & .86 & .85 & .89 & 1 & .86 & .84 & .86 & .87 & .88 \\
\hline $\mathrm{t}_{6}$ & .98 & .98 & .99 & .97 & .86 & 1 & .93 & .98 & .99 & .98 \\
\hline $\mathrm{t}_{7}$ & .94 & .91 & .94 & .96 & .84 & .93 & 1 & .95 & .93 & .92 \\
\hline $\mathrm{t}_{8}$ & .98 & .96 & .97 & .95 & .86 & .98 & .95 & 1 & .97 & .96 \\
\hline $\mathrm{t}_{9}$ & .97 & .99 & .98 & .92 & .87 & .99 & .93 & .97 & 1 & .98 \\
\hline $\mathrm{t}_{10}$ & .96 & .98 & .97 & .93 & .88 & .98 & .92 & .96 & .98 & 1 \\
\hline
\end{tabular}

For $\alpha=0.8$ (given by decision maker), we can see from the above two tables, that for any pair of tuples $t_{p} \& t_{q}$ if $S E\left(t_{p}[U A], t_{q}[U A]\right) \geq \alpha$ then it is also the case that $S E\left(t_{p}[L C], t_{q}[L C]\right) \geq \alpha$

So, we can say that the $\alpha-\operatorname{nfd} U A \stackrel{n f d}{\longrightarrow} L C$ holds for the neutrosophic relational instance $r$. Also, from above Table 2

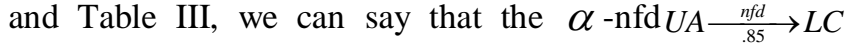

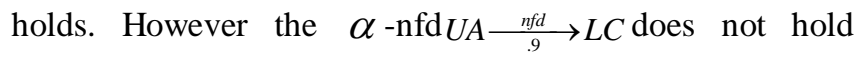
because for tuples $t_{4}$ and $t_{2}, S E\left(t_{4}[U A], t_{2}[U A]\right)=0.91 \geq 0.9$, but $S E\left(t_{4}[L C], t_{2}[L C]\right)=0.87 \leq 0.9$.

\section{Inference Rules for $\alpha-n f d$}

It is well known that in classical relational databases, functional dependencies satisfy a set of inference rules called Armstrong's axioms. In this section, we have derived a set of inference rules for our proposed $\alpha$-nfd. These neutrosophic inference rules are similar to Armstrong's axioms for functional dependency. We call them neutrosophic Armstrong's axiomsand are given as follows:

(A) $\alpha$-nfd reflexive rule

If $Y \subseteq X \subseteq R$, then $X \underset{n f d}{\alpha} Y$

$\left(\mathrm{A}_{2}\right) \alpha$-nfd augmentation rule

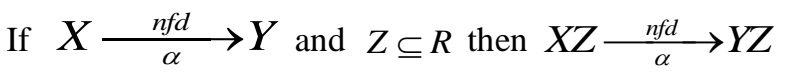

(A3) $\alpha$-nfd transitive rule

If $X \underset{\alpha_{1}}{\stackrel{n f d}{\longrightarrow}} Y, X \underset{\alpha_{2}}{\stackrel{n f d}{\longrightarrow}} Y$ then $X \underset{\min \left(\alpha_{1}, \alpha_{2}\right)}{\stackrel{n f d}{\longrightarrow}} Z$

Theorem 1:Neutrosophic Armstrong's axioms (A1) - (A3) are sound.

Proof:

(A1) $\alpha$-nfd reflexive rule: If $Y \subseteq X \subseteq R$, then $X \underset{\alpha}{\stackrel{n f d}{\rightarrow} Y}$

Let $t_{1}[X](N E)_{\alpha} t_{2}[X]$ is true, i.e., $S E\left(t_{1}\left[X_{i}\right], t_{2}\left[X_{i}\right]\right) \geq \alpha$ $\forall X_{i} \in X$.

Then, $\quad S E\left(t_{1}\left[X_{i}\right], t_{2}\left[X_{i}\right]\right) \geq \alpha \forall X_{i} \in Y \quad$ holds i.e., $t_{1}[Y](N E)_{\alpha} t_{2}[Y]$ is also true.

This implies $X \underset{\alpha}{\stackrel{n f d}{\longrightarrow}} Y$ holds. Hence proved

(A2) $\alpha$-nfd augmentation rule

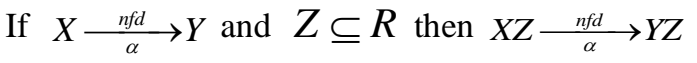

Let $X \underset{\alpha}{\stackrel{n f d}{\longrightarrow}} Y$

Now, from Definition 3.3, for any two tuples $t_{l}$ and $t_{2}$ if $t_{1}[X](N E)_{\alpha} t_{2}[X] \ldots(1)$ is true,

then $t_{1}[Y](N E)_{\alpha} t_{2}[Y] \ldots .(2)$ is also true. Next, suppose $t_{1}[X Z](N E)_{\alpha} t_{2}[X Z] \ldots \ldots .(3)$ is true. This

implies,

$$
S E\left(t_{1}\left[X_{i}\right], t_{2}\left[X_{i}\right]\right) \geq \alpha, \forall X_{i} \in X Z
$$

$S E\left(t_{1}\left[X_{i}\right], t_{2}\left[X_{i}\right]\right) \geq \alpha, \forall X_{i} \in X$ and

$S E\left(t_{1}\left[X_{i}\right], t_{2}\left[X_{i}\right]\right) \geq \alpha, \forall X_{i} \in Z$

now $\operatorname{SE}\left(t_{1}\left[X_{i}\right], t_{2}\left[X_{i}\right]\right) \geq \alpha$

,$\forall X_{i} \in Z \Rightarrow$

$t_{1}[Z](N E)_{\alpha} t_{2}[Z]$

Then from (2) and (4), we get 


$$
t_{1}[Y Z](N E)_{\alpha} t_{2}[Y Z]
$$

Thus, for any two tuples $t_{1}$ and $t_{2}$ if $t_{1}[X Z](N E)_{\alpha} t_{2}[X Z]$ then it is also the case that $t_{1}[Y Z](N E)_{\alpha} t_{2}[Y Z]$ which implies $X Z \underset{\alpha}{\stackrel{n f d}{\longrightarrow}} Y Z$.Hence proved.

(A3) $\alpha$-nfd transitive rule

$$
\begin{aligned}
& \text { If } X \underset{\alpha_{1}}{\stackrel{n f d}{\longrightarrow}} Y, X \underset{\alpha_{2}}{\stackrel{n f d}{\longrightarrow}} Y \text { then } \\
& X \underset{\min \left(\alpha_{1}, \alpha_{2}\right)}{\longrightarrow} Z
\end{aligned}
$$

Let us assume that both the nfds $X \underset{\alpha_{1}}{\stackrel{n f d}{\longrightarrow}} Y, Y \stackrel{n f d}{\underset{\alpha_{2}}{\longrightarrow}} Z$ hold in the relation $r(R)$.

\section{Case I}

$\alpha_{1} \geq \alpha_{2}$ so that $\min \left(\alpha_{1}, \alpha_{2}\right)=\alpha_{2}$.

Given that $X \underset{\alpha_{1}}{\stackrel{n f d}{\longrightarrow}} Y$ and $0 \leq \alpha_{2} \leq \alpha_{1} \leq 1$.

So, using

Proposition 2: we get $X \underset{n f d}{\alpha_{2}} Y$-------- (1)

Then, from (i) we can write $t_{1}[X](N E)_{\alpha_{2}} t_{2}[X] \Rightarrow t_{1}[Y](N E)_{\alpha_{2}} t_{2}[Y] \cdots \ldots .(2)$

Again, since $Y \stackrel{n f d}{\underset{\alpha_{2}}{\longrightarrow}} Z$ holds, so we have

$t_{1}[Y](N E)_{\alpha_{2}} t_{2}[Y] \Rightarrow t_{1}[Z](N E)_{\alpha_{2}} t_{2}[Z] \cdots \ldots .(3)$

Combining (2) and (3), we get

$t_{1}[X](N E)_{\alpha_{2}} t_{2}[X] \Rightarrow t_{1}[Z](N E)_{\alpha_{2}} t_{2}[Z]$ which implies $X \underset{\alpha_{2}}{\stackrel{n f d}{\longrightarrow}} Z$

Hence for $\alpha_{2}=\min \left(\alpha_{1}, \alpha_{2}\right)$, if $X \underset{\alpha_{1}}{\stackrel{n f d}{\longrightarrow}} Y, Y \underset{\alpha_{2}}{\longrightarrow} Z$ then $X \underset{\alpha_{2}}{\stackrel{n f d}{\longrightarrow}} Z$

Case II: $\alpha_{2} \geq \alpha_{1}$ follows similarly. Hence proved.

Using the above neutrosophic Armstrong's axioms, the following results are also derived for $\alpha$-nfd.

(A4) $\alpha$-nfd decomposition rule

If $X \stackrel{n f d}{\stackrel{n}{\longrightarrow}} Y Z$ then $X \underset{\alpha}{\stackrel{n f d}{\longrightarrow}} Y, X \underset{\alpha}{\stackrel{n f d}{\longrightarrow}} Z$

Proof: Given that $X \stackrel{n f d}{\longrightarrow} Y Z \ldots .$. (1)

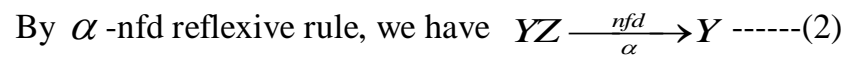

From (1) and (2) using $\alpha$-nfd transitive rule, we get $X \underset{\alpha}{\stackrel{n f d}{\longrightarrow}} \boldsymbol{Y}, X \underset{\alpha}{\stackrel{n f d}{\longrightarrow}} Z$ also follows similarly.

Hence Proved.

(A5) $\alpha$-nfd union rule

If $X \underset{\alpha_{1}}{\stackrel{n f d}{\longrightarrow}} Y, X \underset{\alpha_{2}}{\stackrel{n f d}{\longrightarrow}} Z$ then $X \underset{\min \left(\alpha_{1}, \alpha_{2}\right)}{\longrightarrow} Y Z$

Proof:

Given that $X \underset{\alpha_{1}}{\stackrel{n f d}{\longrightarrow}} Y$----- (1) and $X \underset{\alpha_{2}}{\stackrel{n f d}{\longrightarrow}} Z$------ (2)
From (1) we may write $X \underset{\alpha_{1}}{\stackrel{n f d}{\longrightarrow}} X Y$------- (3)(using $\alpha-$ nfd augmentation rule).

Similarly, from (2) we can write $X Y \underset{\alpha_{2}}{\stackrel{n f d}{\longrightarrow}} Y Z$

Thus from (3) and (4) using $\alpha$-nfd transitive rule, we get $X \underset{\min \left(\alpha_{1}, \alpha_{2}\right)}{\longrightarrow} Y Z$.Hence proved.

(A6) $\alpha$-nfd pseudo transitive rule

If $X \underset{\alpha_{1}}{\stackrel{n f d}{\longrightarrow}} Y$ and $W Y \underset{\alpha_{2}}{\stackrel{n f d}{\longrightarrow}} Z$ then

$W X \underset{\min \left(\alpha_{1}, \alpha_{2}\right)}{\longrightarrow} Z$

Proof: Given that

$$
X \underset{\alpha_{1}}{\stackrel{n f d}{\longrightarrow}} Y \ldots
$$

$W Y \underset{\alpha_{2}}{\stackrel{n f d}{\longrightarrow}} Z$

From (1), using $\alpha$-nfd augmentation rule we can write $W X \underset{\alpha_{1}}{\stackrel{n f d}{\longrightarrow}} W Y$

From (3) and (2) using $\alpha$-nfd transitive rule, we get $W X \underset{\min \left(\alpha_{1}, \alpha_{2}\right)}{\longrightarrow} Z$. Hence proved.

\section{Partial Neutrosophic Functional Dependency}

After validation of Armstrong's axioms in the neutrosophic environment with our present notion of $\alpha$-nfd, let us define partial neutrosophic functional dependency (partial $\alpha$-nfd) as follows:

Definition 4: $Y$ is called partially vague functionally dependent on $X$ at $\alpha$-level of choice, i.e., $X \underset{\alpha}{\stackrel{n f d}{\rightarrow} Y}$ partially, if $X \stackrel{n f d}{\stackrel{n}{\longrightarrow}} Y$, hold and also there exists a nonempty set $X^{\prime} \subset X$, such that, $X \stackrel{n f d}{\rightarrow} Y$. The notation of partial $\alpha$-nfd is needed to define neutrosophic key.

Example 3: Let the relational schema be $\mathrm{R}=\{\mathrm{A}, \mathrm{B}, \mathrm{C}, \mathrm{D}, \mathrm{E}\}$ and the set of $\operatorname{nfds} N$ on $\mathrm{R}$ be given by $N=\{A B C \underset{0.75}{\stackrel{n f f}{\longrightarrow}} D, A C \underset{0.8}{\stackrel{n f d}{\longrightarrow}} D\}$

Then it easily observed that the $\operatorname{nfd} A B C \stackrel{n f d}{0.75} D$ is a partial $\alpha$-nfd.

\section{E. Neutrosophic Key}

In classical relational database, key is a special case of functional dependency. The concept of classical key in theneutrosophic environment to define neutrosophic key with $\alpha$-level of choice where 0,1 is a choice parameter defined by the database designer. A formal definition of neutrosophic keyis as follows:

Definition 5: Let $K \subseteq R_{l}$ and $N$ be a set of nfds for $R_{l}$. Then, $K$ is called a neutrosophic key of $R_{l}$ at $\alpha$-level of choice 
where 0,1 iff $K \underset{\alpha}{\stackrel{n f d}{\longrightarrow}} R_{1} \in N$ and $K \underset{\alpha}{\stackrel{n f d}{\longrightarrow}} R_{1}$ is not a partial $\alpha-$ nfd.

Example 4:Let us assume a relation schema $R_{l}=\left(A_{l}, B_{l}\right.$, $\left.C_{l}, D_{l}\right)$ and a set of nfds

$$
N=\left\{A \underset{0.75}{\stackrel{n f d}{\longrightarrow}} B, A \underset{0.8}{\stackrel{n f d}{\longrightarrow}} C, A \underset{0.7}{\stackrel{n f d}{\longrightarrow} D} D \text { of } R_{l}\right. \text {. }
$$

Find a neutrosophickey of $R_{l}$.

Solution:

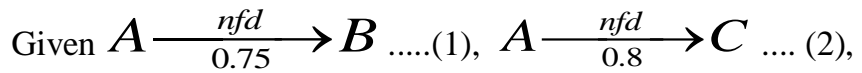

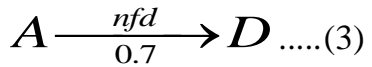

Applying $\alpha$-nfd union rule on (1) and (2), we get $A \underset{n f d}{0.75} \rightarrow B C$

Again, applying $\alpha$-nfd union rule on (3) and (4), we get $A \stackrel{n f d}{\stackrel{0.7}{\longrightarrow}} B C D$

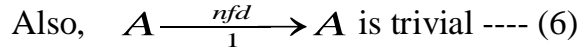

Thus from (5) and (6) using $\alpha$-nfd union rule, we get $A \stackrel{n f d}{\stackrel{0.7}{\longrightarrow}} A B C D$ that is $A \stackrel{n f d}{\rightarrow} R_{1}$ which implies that $A$ is a neutrosophic key of $R_{l}$ at 0.7 -level of choice.

\section{CONCLUSION}

In this paper, we have shown an extension of the classical relational database model with the concepts of neutrosophic set theory, a generalized version of vague sets. We mainly focused on the study of functional dependency in neutrosophic relational database. For this purpose, we have introduced a new kind of neutrosophic functional dependency (called $\alpha$-nfd) based on the idea of $\alpha$ equality of tuples and similarity measure of neutrosophic sets. We also expressed the neutrosophic inference rules and defined partial $\alpha$-nfd and neutrosophic key.

The work may be extended to study Multivalued Dependency and Normalization using $\alpha$-nfd which constitute an important part of a relational database design.

\section{REFERENCES}

[1] F. Smarandache, "First International Conference on Neutrosophy, Neutrosophic Probability, Set, and Logic", University of New Mexico, Vol. 1, No. 3, 2001.

[2] F. Zhao, Z. M. Ma and L. Yan, "A Vague Relational Model and Algebra", Fourth International Conference on Fuzzy Systems and Knowledge Discovery (FSKD2007), Vol. 1, pp. 81-85, 2007.

[3] F. Zhao and Z. M. Ma, "Vague Query Based on Vague Relational Model", Springer- Verlag Berlin Heidelberg, AISC 61, pp. 229-238, 2009.

[4] Deli and S. Broumi, "Neutrosophic soft relations and some properties", Annals of Fuzzy Mathematics and Informatics, Vol. 9, No. 1, pp. 169-182, 2015.

[5] J. Mishra and S. Ghosh, "A New Functional Dependency in a Vague Relational Database Model", International Journal of Computer Applications, Vol. 39, No. 8, pp. 29-33, 2012.

[6] J. Mishra , S. Ghosh, "A Vague Multivalued Data Dependency", International Journal of Fuzzy Information and Engineering, Springer, Vol. 5, No. 4, pp. 459-473,2013.

[7] J. Mishra and S. Ghosh, "Uncertain Query Processing using Vague Sets or Fuzzy Set: Which one isBetter?", International Journal of Computers, Communications and Control (IJCCC), Vol. 9, No. 6, pp. 730-740, 2014

[8] M. Arora and R. Biswas, "Deployment of Neutrosophic technology to retrieve answers for queries posed in natural language", in 3rd International Conference on Computer Science and Information Technology (ICCSIT 2010), Vol. 3, pp. 435-439, 2010.

[9] M. Arora, R. Biswas and U.S. Pandy, "Neutrosophic Relational Database Decomposition", International Journal of Advanced Computer Science and Applications, Vol. 2, No. 8, pp.121-125, 2011.

[10] S. Broumi, "Generalized Neutrosophic Soft Set", International Journal of Computer Science, Engineering and Information Technology, Vol. 3, No. 2, pp. 17-30, 2013.

[11] S. De and J. Mishra "Handle Inconsistent Data using New Method of Neutrosophic Logic for Proper Outcome", International Journal of Science, technology and Management,Vol. 6, No. 2, pp. 383-387, 2017.

[12] S. De and J. Mishra "Query Processing of Inconsistent Data using NeutrosophicSet", IEEE International Conference on Computing Communication and Automation, Vol. 10, No. 6, pp. 120-124, 2016.

[13] S. De and J. Mishra "Compare Different Similarity Measure Formula Based Imprecise Query on Neutrosophic Data", International Conference on Advanced Computing and Intelligent Engineering, Vol.5, No.12, pp. 330-334, 2016.

[14] S. De and J. Mishra "Inconsistent Data Processing Using Vague Set and Neutrosophic Set for Justifying Better Outcome", IEEE International Conference on Inventive Communication and Computational Technologies, Vol.7, No. 4, pp. 26-30, 2017.

[15] S. De and J. Mishra "Processing of Inconsistent Neutrosophic Data and Selecting Primary Key from the Relation" International Conference on Inventive Computing and Informatics, Vol.6, No. 7, pp. 245-250, 2017.

[16] W. L. Gau and D. J. Buehrer, "Vague Sets", IEEE Trans. Syst. Man, Cybernetics, Vol. 23, No. 2, pp.610- 614,1993. 